CORRECTION

\title{
Correction: PPanGGOLiN: Depicting microbial diversity via a partitioned pangenome graph
}

\author{
Guillaume Gautreau, Adelme Bazin, Mathieu Gachet, Rémi Planel, Laura Burlot, \\ Mathieu Dubois, Amandine Perrin, Claudine Médigue, Alexandra Calteau, \\ Stéphane Cruveiller, Catherine Matias, Christophe Ambroise, Eduardo P. C. Rocha, \\ David Vallenet
}

There is an error in S3 File, "List of GenBank assembly accessions for the 439 studied species." The list of assembly accessions was truncated. Please see the complete file below:

\section{Supporting information}

S3 File. List of GenBank assembly accessions for the $\mathbf{4 3 9}$ studied species. This is a TSV file where each line corresponds to all the GenBank assembly accession used in this study for each 'species id' in the NCBI taxonomy.

(TSV)

\section{Reference}

1. Gautreau G, Bazin A, Gachet M, Planel R, Burlot L, Dubois M, et al. (2020) PPanGGOLiN: Depicting microbial diversity via a partitioned pangenome graph. PLoS Comput Biol 16(3): e1007732. https://doi. org/10.1371/journal.pcbi.1007732 PMID: 32191703

G OPEnACCESS

Citation: Gautreau G, Bazin A, Gachet M, Planel R, Burlot L, Dubois M, et al. (2021)

Correction: PPanGGOLiN: Depicting microbial diversity via a partitioned pangenome graph. PLOS Comput Biol 17(12): e1009687. https://doi.org/ 10.1371/journal.pcbi.1009687

Published: December 10, 2021

Copyright: @ 2021 Gautreau et al. This is an open access article distributed under the terms of the Creative Commons Attribution License, which permits unrestricted use, distribution, and reproduction in any medium, provided the original author and source are credited. 